\title{
On mountain pass type algorithms
}

\author{
James Bisgard
}

\begin{abstract}
We consider constructive proofs of the mountain pass lemma, the saddle point theorem and a linking type theorem. In each, an initial "path" is deformed by pushing it downhill using a (pseudo) gradient flow, and, at each step, a high point on the deformed path is selected. Using these high points, a Palais-Smale sequence is constructed, and the classical minimax theorems are recovered. Because the sequence of high points is more accessible from a numerical point of view, we investigate the behavior of this sequence in the final two sections. We show that if the functional satisfies the Palais-Smale condition and has isolated critical points, then the high points form a Palais-Smale sequence, and-passing to a subsequence - the high points will in fact converge to a critical point.

Mathematics Subject Classification (2010). Primary 58E05; Secondary 46T99, 47J30.
\end{abstract}

Keywords. Minimax methods, Critical point theory, Nonlinear functional analysis.

\section{Introduction}

The mountain pass lemma (abbreviated MPL) and the saddle point theorem (SPT) are fundamental tools of nonlinear analysis. Originally, these theorems were proved under the assumption that the corresponding functional satisfied some form of the Palais-Smale (PS) condition. More recent approaches show first the existence of (PS) sequences under the assumptions of the MPL or the SPT, and then show that some subsequence converges. The advantage to this method is that progress has been made in situations where the functional doesn't satisfy the (PS) condition. (See for example, the recent paper [13] and its references.)

The goal of this paper is to present computationally accessible proofs of the MPL, the SPT, and a linking type theorem (LTT). First, we present proofs of versions of the MPL (Sect. 3), the SPT (Sect. 4), and LTT (Sect. 5), without using Ekeland's principle or the deformation lemma. However, it is necessary 
to make an extra assumption about the regularity of the functional $I$ (see LL). The general idea is to take an initial path, push it downhill, pick a high point on this new path and then repeat. Since calculating these high points is in principle straightforward, the behavior of this sequence is important to understand. Under the assumption that $I$ satisfies the (PS) sequence and critical points of $I$ are isolated, we show in Sects. 6 and 7 that this sequence of high points is a (PS) sequence, and by passing to an appropriate subsequence, the high points converge to a critical point.

Throughout, we use a version of the negative gradient flow, although a semi-linear heat flow can be used for the constructive type proofs. An early example of the negative gradient approach is used in $[8,16]$, where solutions of certain types of differential equations are found as elements of the $\omega$-limit set of the negative gradient flow for appropriately picked initial points. In [3] a heat flow is used to show the existence of homoclinic type solutions for a Hamiltonian with two wells at different levels, while in [5], a heat flow is used to prove the basic deformations for the MPL. More recently, Rabinowitz and Bolotin have used a heat flow for functionals that satisfy the (PS) condition in cones, in $[4,15]$.

There has been much recent work on numerically computing critical points arising from the MPL. One of the first examples is the mountain pass algorithm of Choi and McKenna [6]. There, the initial path is a line segment, and a new path is found by moving the high point from the original line downhill and considering a line segment connecting 0 and the deformed high point. This process is repeated until the gradient at the high point is small. Unfortunately, there is no guarantee that the values of the functional on the new line are everywhere smaller than the values on the original, and the algorithm may not converge. More recently, in [1], Barutello and Terracini proposed an algorithm for the MPL: given an initial curve $\gamma$, their algorithm constructs a sequence of points $y_{n}$ that converge to a mountain pass type point. However, the algorithm needs to minimize the size of the gradient along a flow line. In comparison, finding a sequence of high points is more computationally accessible. In addition, the methods here apply to the SPT and a LTT. Lewis and Pang [10] used level set methods to detect critical points of mountain pass type. By minimizing distances between different components of sub-level sets, Lewis and Pang are able to prove very useful convergence rates, but such algorithms may be more difficult to implement. For critical points with higher Morse index, Ding et al. in [7] and later Li and Zhou in [11,12] and Wang and Zhou in [17] proposed algorithms based on a local linking, although we find the ideas here to be more natural.

\section{Pseudo-gradients and flows}

Suppose that $E$ is a Banach space, and $I \in C^{1}(E, \mathbb{R})$. Let $\mathcal{K}:=\{x \in E$ : $\left.I^{\prime}(x)=0\right\}$. It is well known ([9] or [14]) that there exists a locally Lipschitz function $V: E \backslash \mathcal{K} \rightarrow E$ such that 


$$
\begin{array}{ll}
\text { (PG1) } & \|V(x)\| \leq 2\left\|I^{\prime}(x)\right\| \\
\text { (PG2) } & I^{\prime}(x) V(x) \geq\left\|I^{\prime}(x)\right\|^{2} .
\end{array}
$$

Note that (PG2) implies that if $V(x)=0$, then $I^{\prime}(x)=0$. Thus, in fact, $V: E \backslash \mathcal{K} \rightarrow E \backslash\{0\}$. Since $V$ is locally Lipschitz, so too is $\frac{1}{1+\|V(x)\|}$. Therefore,

$$
\begin{aligned}
\frac{d}{d t} \varphi_{t}(x) & =-\frac{V\left(\varphi_{t}(x)\right)}{1+\left\|V\left(\varphi_{t}(x)\right)\right\|} \\
\varphi_{0}(x) & =x
\end{aligned}
$$

defines a local flow $\varphi_{t}$ on $E \backslash \mathcal{K}$. (Note that if $E$ is a Hilbert space, we may use the gradient $\nabla I$ in place of $V$.) If $\varphi_{t}(x)$ remains away from $\mathcal{K}$, then $\varphi_{t}(x)$ will exist for all $t \in \mathbb{R}$, since the right hand side of (2.1) is bounded. We next investigate how $\varphi_{t}(x)$ can approach $\mathcal{K}$. Since $V$ is bounded, if there is a sequence $t_{n} \rightarrow t_{0}$ such that $\varphi_{t_{n}}(x) \rightarrow u \in \mathcal{K}$, we may in fact conclude that $\varphi_{t}(x) \rightarrow u$ as $t \rightarrow t_{0}$. To eliminate the possibility that $\varphi_{t}(x) \rightarrow u \in \mathcal{K}$ in finite time, we assume

$$
\begin{aligned}
& \text { for each } x \in \mathcal{K} \text {, there is an } R>0 \text { and an } M>0 \text { such } \\
& \text { that }\left\|I^{\prime}(u)-I^{\prime}(v)\right\| \leq M\|u-v\| \text { for all } u, v \in B_{R}(x) .
\end{aligned}
$$

Assumption (LL) says that for each $u \in \mathcal{K}$, there is a ball around $u$ on which $I^{\prime}$ is Lipschitz. Thus, any functional $I$ whose derivative is locally Lipschitz will satisfy (LL). In particular, any $C^{2}$ functional will satisfy (LL). As another example, if $V \in C^{2}\left(\mathbb{R} \times \mathbb{R}^{n}, \mathbb{R}\right)$, then $I(u):=\int_{a}^{b} \frac{1}{2}|\dot{u}(t)|^{2}-V(t, u(t)) d t$ is a $C^{1}$ functional on $W^{1,2}([a, b])$, whose derivative is locally Lipschitz. If it is also assumed that $V$ is 1-periodic in $t, V(t, 0)=0<V(t, x)$ for all $x \in \mathbb{R}^{n} \backslash\{0\}$, $V(t, 0)$ is a non-degenerate local maximum for each $t$, and $\liminf _{|x| \rightarrow \infty} V(t, x)=$ $-\alpha<0$ for all $t \in[0,1]$, then $I(u):=\int_{\mathbb{R}} \frac{1}{2}|\dot{u}(t)|^{2}-V(t, u(t)) d t$ is a $C^{1}$ functional on $W^{1,2}(\mathbb{R})$ whose derivative is locally Lipschitz (see for example [2]). We now show that, assuming (LL), if $x \notin \mathcal{K}$, then $\varphi_{t}(x)$ cannot reach $\mathcal{K}$ in finite time.

Lemma 2.1. Suppose that $I \in C^{1}(E, \mathbb{R})$ and $I$ satisfies $(\mathrm{LL})$. If $x \notin \mathcal{K}, \varphi_{t}(x)$ is defined by (2.1), and $\varphi_{t}(x) \rightarrow u \in \mathcal{K}$ as $t \rightarrow t_{0}$, then $t_{0}=+\infty$.

Proof. Let us suppose that there is in fact an $x \notin \mathcal{K}$ and a $t_{0} \in(0, \infty)$ such that $\varphi_{t}(x) \rightarrow u \in \mathcal{K}$ as $t \rightarrow t_{0}$. Since $\varphi_{t}(x) \rightarrow u$, we may assume that there is a $t^{\star}$ such that if $t \in\left(t^{\star}, t_{0}\right)$, then $\varphi_{t}(x) \in B_{R}(u)$, where $R$ is from assumption (LL). Now, let

$$
g(x):=-\frac{V(x)}{1+\|V(x)\|} \quad \text { for } x \in B_{R}(u)
$$


and $g(u):=0$. Note that $g$ is then continuous on $B_{R}(u)$ and for $x \in B_{R}(u)$, we have

$$
\begin{aligned}
\|g(x)\| & \leq \frac{\|V(x)\|}{1+\|V(x)\|} \\
& \leq\|V(x)\| \\
& \leq 2\left\|I^{\prime}(x)\right\| \quad(\text { by }(\mathrm{PG} 1)) \\
& =2\left\|I^{\prime}(x)-I^{\prime}(u)\right\| \quad(\text { since } u \in \mathcal{K}) \\
& \leq 2 M\|x-u\|,
\end{aligned}
$$

by (LL). Since $\varphi_{t}(x) \in B_{R}(u)$ for all $t \in\left(t^{\star}, t_{0}\right)$, (2.1) then implies for all $s, t \in\left(t^{\star}, t_{0}\right)$ with $s \leq t$ that

$$
\begin{aligned}
\left\|\varphi_{t}(x)-\varphi_{s}(x)\right\| & =\left\|\int_{s}^{t} g\left(\varphi_{\tau}(x)\right) d \tau\right\| \\
& \leq \int_{s}^{t}\left\|g\left(\varphi_{\tau}(x)\right)\right\| d \tau \\
& \leq 2 M \int_{s}^{t}\left\|\varphi_{\tau}(x)-u\right\| d \tau .
\end{aligned}
$$

Letting $t \rightarrow t_{0}$, the inequality above implies for all $s \in\left(t^{\star}, t_{0}\right)$ that

$$
\left\|\varphi_{s}(x)-u\right\| \leq 2 M \int_{s}^{t_{0}}\left\|\varphi_{\tau}(x)-u\right\| d \tau .
$$

Gronwall's Inequality then implies that $\varphi_{s}(x)=u$ for all $x \in\left(t^{\star}, t_{0}\right)$, which is impossible.

By Lemma 2.1, (2.1) defines a flow on $E \backslash \mathcal{K}$. We now define a flow on all of $E$. Inequalities (PG1) and (PG2) imply that $\left\|I^{\prime}(x)\right\| \leq\|V(x)\| \leq 2\left\|I^{\prime}(x)\right\|$, and so if $x_{n} \rightarrow x \in \mathcal{K}$, then $V\left(x_{n}\right) \rightarrow 0$, and so we may think of $V$ as being extended to be 0 at critical points of $I$. Thus, if $x \in \mathcal{K}$, we would expect the flow to leave $x$ fixed, and so we define our flow to fix points in $\mathcal{K}$. We now define $\psi_{t}: \mathbb{R} \times E \rightarrow E$ by

$$
\psi_{t}(x):= \begin{cases}x & \text { if } x \in \mathcal{K} \\ \varphi_{t}(x) & \text { if } x \notin \mathcal{K},\end{cases}
$$

where $\varphi_{t}(x)$ is the unique solution of (2.1). Since the the right side of $(2.1)$ is bounded independent of $t$ and $x$, Lemma 2.1 implies that $\varphi_{t}(x)$ exists for all $t \in \mathbb{R}$. Note that clearly $\psi_{t}\left(\psi_{s}(x)\right)=\psi_{t+s}(x)$ for all $s, t \in \mathbb{R}$ and all $x \in E$. Thus, to verify that $\psi_{t}$ defines a flow, we need to show that $\psi_{t}(x)$ is continuous in $t$ and $x$, which is a consequence of the following lemma.

Lemma 2.2. For every $x \in E$ and every $T>0$, there is a neighborhood $N$ of $x$ and a constant $K$ such that for all $y \in N$, if $\psi_{t}(y) \in N$ for all $t \in[0, T]$, then $\left\|\psi_{t}(x)-\psi_{t}(y)\right\| \leq e^{K T}\|x-y\|$.

Proof. If $x \notin \mathcal{K}$, then we may use standard arguments, relying on the fact that $V$ is locally Lipschitz. Suppose now that $x \in \mathcal{K}$. In this case, take $N=$ $B_{R}(x)$, where $B_{R}(x)$ is the ball specified in assumption (LL). Suppose now that 
$y \in B_{R}(x)$. If $y \in \mathcal{K}$, then $\left\|\psi_{t}(x)-\psi_{t}(y)\right\|=\|x-y\| \leq e^{K T}\|x-y\|$ for any positive $T$. If $y \notin \mathcal{K}$, then

$$
\begin{aligned}
\psi_{t}(x)-\psi_{t}(y) & =x-\varphi_{t}(y) \\
& =x-\left(y+\int_{0}^{t} \frac{d}{d x} \varphi_{s}(y) d s\right) \\
& =x-y+\int_{0}^{t} \frac{V\left(\varphi_{s}(y)\right)}{1+\left\|V\left(\varphi_{s}(y)\right)\right\|} d s .
\end{aligned}
$$

Therefore, by (PG1),

$$
\begin{aligned}
\left\|\psi_{t}(x)-\psi_{t}(y)\right\| & \leq\|x-y\|+\int_{0}^{t}\left\|V\left(\varphi_{s}(y)\right)\right\| d s \\
& \leq\|x-y\|+\int_{0}^{t} 2\left\|I^{\prime}\left(\varphi_{s}(y)\right)\right\| d s \\
& =\|x-y\|+\int_{0}^{t} 2\left\|I^{\prime}\left(\varphi_{s}(y)\right)-I^{\prime}\left(\psi_{s}(x)\right)\right\| d s
\end{aligned}
$$

since $\psi_{s}(x)=x$ for all $s$ and $I^{\prime}(x)=0$. By assumption (LL), there is an $R>0$ and a $M>0$ such that if $u, v \in B_{R}(x)$, then $\left\|I^{\prime}(u)-I^{\prime}(v)\right\| \leq M\|u-v\|$. Therefore, if $\varphi_{s}(y) \in B_{R}(x)$ for $s \in[0, t]$, then we have $\left(\right.$ since $\varphi_{s}(y)=\psi_{s}(y)$ by definition of $\psi$ )

$$
\left\|\psi_{t}(x)-\psi_{t}(y)\right\| \leq\|x-y\|+2 M \int_{0}^{t}\left\|\psi_{s}(y)-\psi_{s}(x)\right\| d s .
$$

If $g(t):=\left\|\psi_{t}(x)-\psi_{t}(y)\right\|$, then $g$ is continuous, and the inequality above implies that

$$
g(t) \leq g(0)+2 M \int_{0}^{t} g(s) d s
$$

But then, by Gronwall's Inequality, we have $g(t) \leq g(0) e^{2 M t}$. Therefore, we will have

$$
\left\|\psi_{t}(x)-\psi_{t}(y)\right\| \leq e^{2 M t}\|x-y\|,
$$

which is the desired statement.

Lemma 2.2 implies that $\psi_{t}(x)$ is continuous in $t$ and $x$, and in particular $\psi_{t}(x)$ defines a flow on $E$. Notice also that in our proofs of Lemmas 2.1 and 2.2 , we did not use the full strength of (LL), but rather only the following slightly weaker version:

for each $u \in \mathcal{K}$, there is an $R>0$ and an $M>0$ such that $\left\|I^{\prime}(x)\right\| \leq M\|x-u\|$ for all $x \in B_{R}(u)$.

That is, we use only the fact that the size of the derivative grows at most linearly in a neighborhood of the critical point. Without an assumption of 
this type, it is unclear how to define a flow everywhere on $E$. One seemingly natural possibility is to define

$$
\psi_{t}(x)= \begin{cases}\varphi_{t}(x) & \text { if } t \leq t_{0} \\ \lim _{t \rightarrow t_{0}} \varphi_{t}(x) & \text { if } t>t_{0},\end{cases}
$$

where $\left(0, t_{0}\right)$ is the maximal interval on which $\varphi_{t}(x)$ is defined. However, this definition does not produce a flow, since flow lines cannot intersect. In the traditional deformation lemma approach, the problem with the behavior of the flow close to the critical points is avoided by using locally Lipschitz cutoff functions that vanish in a neighborhood of critical points. Typically, these functions are expressed in terms of distance from subsets of critical points. From a computational point of view, calculating the value of such cut-off functions is difficult, because their calculation necessitates knowing the position of the critical points.

Lemma 2.3. For any $x \in E, f(t):=I\left(\psi_{t}(x)\right)$ is non-increasing.

Proof. This is clear if $x \in \mathcal{K}$. Suppose next that $x \notin \mathcal{K}$, and so $\psi_{t}(x)=\varphi_{t}(x)$. By the chain rule, we have

$$
\begin{aligned}
f^{\prime}(t) & =I^{\prime}\left(\varphi_{t}(x)\right) \frac{d}{d t} \varphi_{t}(x) \\
& =-\frac{1}{1+\left\|V\left(\varphi_{t}(x)\right)\right\|} I^{\prime}\left(\varphi_{t}(x)\right) V\left(\varphi_{t}(x)\right) \\
& \leq-\frac{1}{1+\left\|V\left(\varphi_{t}(x)\right)\right\|}\left\|I^{\prime}\left(\varphi_{t}(x)\right)\right\|^{2} \\
& \leq 0,
\end{aligned}
$$

by (PG2).

Proposition 2.4. For every $x \in E$ and $\alpha \in \mathbb{R}$, if $I\left(\psi_{t}(x)\right) \geq \alpha$ for all $t \geq 0$, then there is a sequence $\left(t_{n}\right)$ with $t_{n} \rightarrow \infty$ such that $I\left(\psi_{t_{n}}(x)\right) \geq \alpha$ is bounded and $I^{\prime}\left(\psi_{t_{n}}(x)\right) \rightarrow 0$ as $n \rightarrow \infty$.

Proof. Again, this is clear if $x \in \mathcal{K}$. Suppose then that $x \notin \mathcal{K}$. Then $\psi_{t}(x)=$ $\varphi_{t}(x)$. By Lemma 2.3 and the assumption that $I\left(\psi_{t}(x)\right) \geq \alpha, \lim _{t \rightarrow \infty} I\left(\varphi_{t}(x)\right)$ exists. We have

$$
\begin{aligned}
I\left(\varphi_{t}(x)\right)-I(x) & =\int_{0}^{t} \frac{d}{d s} I\left(\varphi_{s}(x)\right) d s \\
& =\int_{0}^{t} I^{\prime}\left(\varphi_{s}(x)\right) \frac{d}{d s} \varphi_{s}(x) d x \\
& =\int_{0}^{t}-\frac{1}{1+\left\|V\left(\varphi_{s}(x)\right)\right\|} I^{\prime}\left(\varphi_{s}(x)\right) V\left(\varphi_{s}(x)\right) d s \\
& \leq \int_{0}^{t}-\frac{1}{1+\left\|V\left(\varphi_{s}(x)\right)\right\|}\left\|I^{\prime}\left(\varphi_{s}(x)\right)\right\|^{2} d x
\end{aligned}
$$


by (PG2). By (PG1), 2\|I'(x)\| $\geq\|V(x)\|$, and so $\left\|I^{\prime}(x)\right\|^{2} \geq \frac{1}{4}\|V(x)\|^{2}$. Therefore,

$$
I(x)-I\left(\varphi_{t}(x)\right) \geq \int_{0}^{t} \frac{1}{4} \frac{\left\|V\left(\varphi_{s}(x)\right)\right\|^{2}}{1+\left\|V\left(\varphi_{s}(x)\right)\right\|} d s .
$$

Since $\lim _{t \rightarrow \infty} I\left(\varphi_{t}(x)\right)$ exists, taking $t \rightarrow \infty$ in the inequality above, we see that

$$
\int_{0}^{\infty} \frac{\left\|V\left(\varphi_{s}(x)\right)\right\|^{2}}{1+\left\|V\left(\varphi_{s}(x)\right)\right\|} d s<\infty .
$$

Therefore, there is a sequence $\left(t_{n}\right)$ with $t_{n} \rightarrow \infty$ such that $\frac{\left\|V\left(\varphi_{t_{n}}(x)\right)\right\|^{2}}{1+\left\|V\left(\varphi_{t_{n}}(x)\right)\right\|} \rightarrow 0$. This implies that $\left\|V\left(\varphi_{t_{n}}(x)\right)\right\| \rightarrow 0$. Note that (PG2) implies that if $I^{\prime}(x) \neq 0$, then $\|V(x)\| \geq\left\|I^{\prime}(x)\right\|$. Thus, $\left\|I^{\prime}\left(\varphi_{t_{n}}(x)\right)\right\| \rightarrow 0$. Thus, the sequence $\left(\varphi_{t_{n}}(x)\right)$ is a (PS) sequence for $I$. Since $\lim _{t \rightarrow \infty} I\left(\varphi_{t}(x)\right)$ exists and $I\left(\varphi_{t}(x)\right) \geq \alpha$, we must also have $I\left(\varphi_{t_{n}}(x)\right) \geq \alpha$, and so the sequence $\left(\varphi_{t_{n}}(x)\right)$ satisfies the requirements.

\section{The mountain pass lemma}

Suppose now that $I$ satisfies

$(\mathrm{MP} 1) \quad I(0)=0$ and $I^{\prime}(0)=0$

(MP2) there is an $r>0, \alpha>0$ such that $\inf _{x \in \partial B_{r}(0)} I(x) \geq \alpha$

(MP3) there is an $e \notin B_{r}(0)$ with $I(e) \leq 0$.

In [18], Willem showed that in this situation, there is a (PS) sequence $\left(x_{n}\right)$ such that $I\left(x_{n}\right) \rightarrow c$, where

$$
c:=\inf _{h \in \Gamma} \max _{s \in[0,1]} I(h(s)),
$$

and

$$
\Gamma:=\left\{h \in C([0,1] \times E, E): h(0)=0, I(h(1)) \leq 0, \text { and } h(1) \notin B_{r}(0)\right\} .
$$

We prove the following version, which is very similar to Barutello and Terracini's proof in [1]:

Theorem 3.1. Suppose that I satisfies (LL) and (MP1-3). For any $h \in \Gamma$, there is a sequence $\left(t_{n}\right)$ with $t_{n} \rightarrow \infty$ and an $s^{\star} \in[0,1]$ such that $\left(\psi_{t_{n}}\left(h\left(s^{\star}\right)\right)\right)$ is a $(P S)$ sequence with $I\left(\psi_{t_{n}}\left(h\left(s^{\star}\right)\right)\right) \rightarrow \hat{c}$, where $\hat{c} \geq c$.

Proof. Let $h \in \Gamma$. For each $i \in \mathbb{N}$, we claim that $\psi_{i}(h(s)) \in \Gamma$. Notice that since $\psi_{t}(x)$ is continuous in $t$ and $x, \psi_{i}(h(s))$ is continuous. Moreover, $\psi_{t}(0)=0$, since $0 \in \mathcal{K}$. Next, Lemma 2.3 implies that $I\left(\psi_{i}(h(1))\right) \leq I(h(1)) \leq 0$. To show that $\psi_{i}(h(1)) \notin B_{r}(0)$, note that if $\psi_{i}(h(1)) \in B_{r}(0)$, there would have to be a $\tau \in(0, i)$ such that $\psi_{\tau}(h(1)) \in \partial B_{r}(0)$. By (MP2) and the definition of $\Gamma$, that would imply that $I$ doesn't decrease along the flow of $\psi$, contradicting Lemma 2.3. 
For each $i$, there is an $s_{i} \in[0,1]$ so that $I\left(\psi_{i}\left(h\left(s_{i}\right)\right)\right)=\max _{s \in[0,1]} I\left(\psi_{i}(h(s))\right)$. Note that since $\psi_{i}(h(s)) \in \Gamma, I\left(\psi_{i}\left(h\left(s_{i}\right)\right)\right) \geq c$. Since [0,1] is compact, passing to a relabeled subsequence, there is a sequence $\left(s_{i}\right)$ such that $s_{i} \rightarrow s^{\star}$. We now claim that $I\left(\psi_{t}\left(h\left(s^{\star}\right)\right)\right) \geq c$ for all $t \geq 0$. If not, then for some large $j, I\left(\psi_{j}\left(h\left(s^{\star}\right)\right)\right)<c$. But then, for all large $i, I\left(\psi_{j}\left(h\left(s_{i}\right)\right)\right)<c$. We may assume that $i>j$. Then, by Lemma 2.3 , we would have

$$
I\left(\psi_{i}\left(h\left(s_{i}\right)\right)\right)=I\left(\psi_{i-j}\left(\psi_{j}\left(h\left(s_{i}\right)\right)\right)\right) \leq I\left(\psi_{j}\left(h\left(s_{i}\right)\right)\right)<c,
$$

which contradicts the choice of $s_{i}$ so that $I\left(\psi_{i}\left(h\left(s_{i}\right)\right)\right)=\max _{s \in[0,1]} I\left(\psi_{i}(h(s))\right)$.

Proposition 2.4 then implies that the existence of an appropriate sequence $t_{n}$ so that $\left(\psi_{t_{n}}\left(h\left(s^{\star}\right)\right)\right)$ is a (PS) sequence for which $I\left(\psi_{t_{n}}\left(h\left(s^{\star}\right)\right)\right) \geq c$.

Notice that Theorem 3.1 doesn't imply the existence of a (PS) sequence $\left(u_{n}\right)$ for which $I\left(u_{n}\right) \rightarrow c$, where $c$ is specified by the standard minimax formula. However, we have the following

Proposition 3.2. Suppose I satisfies (LL) and (MP1-3). Then there is a (PS) sequence $\left(u_{n}\right)$ such that $I\left(u_{n}\right) \rightarrow c$.

Proof. For every $n \in \mathbb{N}$, pick $h_{n} \in \Gamma$ such that $\max _{s \in[0,1]} I\left(h_{n}(s)\right) \leq c+\frac{1}{n}$. Arguing as in the proof of Theorem 3.1, there is a (PS) sequence $\left(x_{n, j}\right)$ such that $c \leq I\left(x_{n, j}\right) \leq c+\frac{1}{n}$ for all $j \in \mathbb{N}$. Since $\left(x_{n, j}\right)$ is a (PS) sequence, there is a $j_{n}$ such that $\left\|I^{\prime}\left(x_{n, j_{n}}\right)\right\| \leq \frac{1}{n}$. Let $u_{n}:=x_{n, j_{n}}$. Then $I^{\prime}\left(u_{n}\right) \rightarrow 0$ and $I\left(u_{n}\right) \rightarrow c$ as $n \rightarrow \infty$.

A disadvantage of the approach above is the extra regularity assumption on $I$ necessary to ensure that we have a flow on all of $E$. Without (LL), cut-off functions that are 0 in a neighborhood of $\mathcal{K}$ are used to get a flow defined on all of $E$. An advantage of this method is particular choices of initial paths $h$ can be used to find critical points with particular behavior. Notice that the necessary ingredient is a flow that decreases $I$ (Lemma 2.3) and for which some version of Proposition 2.4 holds. In general, any flow that decreases $I$ and whose $\omega$-limit sets are non-empty and consist of critical points of $I$ will work. For example, in [3], a semi-linear heat flow is used together with a particular choice of initial path to find homoclinic solutions of a double-well Hamiltonian equation with wells at different levels.

An advantage of this approach is that it is in a sense constructive: In order to find a (PS) sequence, pick any path in $\Gamma$, push the path downhill using the negative (pseudo)-gradient, keep track of the high points on the deformed path and use those high points to find an initial value $x$ for which $I\left(\psi_{t}(x)\right)$ is bounded from below. This is reminiscent of the idea of some numerical mountain pass algorithms (for example, [6]): given an initial path, deform the path in some fashion and pick the high point on the new path and repeat: Step 1: Pick an $h \in \Gamma$, and set $i=1$.

Step 2: Deform $h$ using the negative gradient flow to $\psi_{i}(h(\cdot))$.

Step 3: Find $s_{i} \in[0,1]$ such that $I\left(\psi_{i}\left(h\left(s_{i}\right)\right)\right)=\max _{s \in[0,1]} I\left(\psi_{i}(h(s))\right)$.

Step 4: Increment $i$, and then return to step 2 . 
We consider the question of convergence of the sequence of high points $\psi_{i}\left(h\left(s_{i}\right)\right)$ in Sects. 6 and 7.

\section{The saddle point theorem}

Suppose now that $I \in C^{1}(E, \mathbb{R})$ satisfies

(SP1) $E=V \oplus X$, where $V$ is finite dimensional

(SP2) there is an $r>0$ and an $\alpha$ such that $\max _{u \in \partial B_{r}(0) \cap V} I(u) \leq \alpha$

(SP3) there is a $\beta>\alpha$ such that $\inf _{u \in X} I(u) \geq \beta$.

The saddle point theorem [14] says that if $I$ satisfies (SP1-3) and the (PS) condition, then

$$
c=\inf _{h \in \Gamma} \max _{x \in \overline{B_{r}(0)} \cap V} I(h(x)) \geq \beta
$$

is a critical value of $I$, where

$$
\Gamma=\left\{h \in C\left(\overline{B_{r}(0)} \cap V, E\right): h=\text { id on } \partial B_{r}(0) \cap V\right\} .
$$

In [18], it is shown under assumptions (SP1-3) that there exists a (PS) sequence $x_{n}$ such that $I\left(x_{n}\right) \rightarrow c$. We take

$$
\begin{array}{r}
\Gamma_{1}=\left\{h \in C\left(\overline{B_{r}(0)} \cap V, E\right): \text { there exists } A_{h} \in C\left([0,1] \times \overline{B_{r}(0)} \cap V, E\right)\right. \\
\text { satisfying properties (a), (b), and (c) }\},
\end{array}
$$

where

(a) $A_{h}(0, x)=x$ for all $x \in \partial B_{r}(0) \cap V$,

(b) $A_{h}(1, x)=h(x)$ for all $x \in \overline{B_{r}(0)} \cap V$,

(c) For each $x \in \partial B_{r}(0) \cap V, t \mapsto I\left(A_{h}(t, x)\right)$ is non-increasing.

We then define

$$
c_{1}=\inf _{h \in \Gamma_{1}} \max _{x \in B_{r}(0) \cap V} I(h(x)) .
$$

We have the following:

Theorem 4.1. Suppose that I satisfies (SP1-3) and (LL). Then $c_{1} \geq \beta$. Moreover, for any $h \in \Gamma$, there is an $s^{\star} \in \overline{B_{r}(0)} \cap V$ and a sequence $\left(t_{n}\right)$ with $t_{n} \rightarrow \infty$ such that if $u_{n}:=\varphi_{t_{n}}\left(h\left(s^{\star}\right)\right)$, then $\left(u_{n}\right)$ is a (PS) sequence and $I\left(u_{n}\right) \geq c_{1}$.

Proof. To show $c_{1} \geq \beta$, it suffices to show that for any $h \in \Gamma_{1}$, there is an $x \in \overline{B_{r}(0)} \cap V$ such that $h(x) \in X$. Let $P: E \rightarrow V$ be the projection onto $V$, and note that $P u=0$ if and only if $u \in X$, and so we need only show there is an $x \in \overline{B_{r}(0)} \cap V$ such that $P h(x)=0$. For any $t \in[0,1], x \in$ $\overline{B_{r}(0)} \cap V$, let $H(t, x)=A_{h}(t, x)$. Since $H(0, x)=P A_{h}(0, x)=x$ for all $x \in \partial B_{r}(0) \cap V$, we have $\operatorname{deg}\left(H(0, x), B_{r}(0) \cap V, 0\right)=\operatorname{deg}\left(\mathrm{id}, B_{r}(0) \cap V, 0\right)=1$. Because $\operatorname{deg}\left(P h, B_{r}(0) \cap V, 0\right)=\operatorname{deg}\left(H(1, x), B_{r}(0) \cap V, 0\right)$, we need only show that $\operatorname{deg}\left(H(1, x), B_{r}(0) \cap V, 0\right)=\operatorname{deg}\left(H(0, x), B_{r}(0) \cap V, 0\right)$. This will follow from the homotopy independence of the degree if we can show there are no 
$(t, x) \in[0,1] \times \partial B_{r}(0) \cap V$ such that $H(t, x)=0$. Suppose there was such a $(t, x)$. Then we would have $P A_{h}(t, x)=0$, which implies that $A_{h}(t, x) \in X$. (c), (SP2) and (SP3) imply that $\beta \leq I\left(A_{h}(t, x)\right) \leq I\left(A_{h}(0, x)\right)=I(x) \leq \alpha$, a contradiction.

Let $h \in \Gamma_{1}$. For each $i \in \mathbb{N}$, we show that $\psi_{i} \circ h \in \Gamma_{1}$. Since $h \in \Gamma_{1}$, there is an $A_{h}$ corresponding to $h$ that satisfies (a-c). We now find an appropriate $\Lambda$ for $\psi_{i} \circ h$. Let

$$
\Lambda(t, x):= \begin{cases}A_{h}(2 t, x) & \text { if } t \in\left[0, \frac{1}{2}\right), x \in \overline{B_{r}(0)} \cap V \\ \psi_{i(2 t-1)}(h(x)) & \text { if } t \in\left[\frac{1}{2}, 1\right], x \in \overline{B_{r}(0)} \cap V .\end{cases}
$$

Note that as $t \nearrow \frac{1}{2}, \Lambda(t, x)=A_{h}(2 t, x) \rightarrow A_{h}(1, x)=h(x)$, and $\Lambda\left(\frac{1}{2}, x\right)=$ $\psi_{0}(h(x))=h(x)$, and so $\Lambda \in C\left([0,1] \times \overline{B_{r}(0)} \cap V, E\right)$. For $($ a $)$, we have $\Lambda(0, x)=$ $A_{h}(0, x)=x$ for all $x \in \partial B_{r}(0) \cap V$. For (b), $\Lambda(1, x)=\psi_{i}(h(x))=\psi_{i} \circ h(x)$ for all $x \in \overline{B_{r}(0)} \cap V$. (c) follows from the corresponding properties of $A_{h}$ and Lemma 2.3. Thus $\psi \circ h \in \Gamma_{1}$.

For each $i$, let $s_{i} \in \overline{B_{r}(0)} \cap V$ be chosen so that

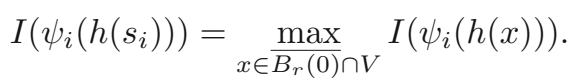

Because $\overline{B_{r}(0)} \cap V$ is compact, there is a subsequence $\left(s_{i_{j}}\right)$ of $\left(s_{i}\right)$ such that $s_{i_{j}} \rightarrow s^{\star} \in \overline{B_{r}(0)} \cap V$. We can make the same argument as in the proof of Theorem 3.1 to get an appropriate (PS) sequence.

We next show that $c_{1}=c$. Since $\Gamma \subseteq \Gamma_{1}$, it is clear that $c_{1} \leq c$. The opposite inequality is an immediate consequence of the following proposition.

Proposition 4.2. For any $h \in \Gamma_{1}$, there is a $\tilde{h} \in \Gamma$ such that

$$
\max _{x \in B_{r}(0) \cap V} I(h(x))=\max _{x \in B_{r}(0) \cap V} I(\tilde{h}(x)) .
$$

Proof. Let $h \in \Gamma_{1}$ be fixed, and suppose that $A_{h}$ satisfies (a-c). We then define

$$
\tilde{h}(x):= \begin{cases}h(2 x) & \text { if } x \in \overline{B_{r / 2}(0)} \cap V \\ A_{h}\left(\frac{2}{r}(r-\|x\|), r \frac{x}{\|x\|}\right) & \text { if } x \in\left(\overline{B_{r}(0)} \backslash B_{r / 2}(0)\right) \cap V .\end{cases}
$$

Note that as $\|x\| \searrow \frac{r}{2}, A_{h}\left(\frac{2}{r}(r-\|x\|), \frac{r x}{\|x\|}\right) \rightarrow A_{h}(1,2 x)=h(2 x)$ by (a). Thus, $\tilde{h}$ is continuous. Next, note that if $\|x\|=r$, then $\tilde{h}(x)=A_{h}(0, x)=x$ and so $\tilde{h} \in \Gamma$. The following immediate consequences of the definition and properties $(\mathrm{a}-\mathrm{c})$ of $A_{h}$ finish the proof:

$$
\underset{x \in \max _{B_{r / 2}(0)} \cap V}{ } I(\tilde{h}(x))=\max _{x \in \overline{B_{r}(0)} \cap V} I(h(x)) \geq \beta
$$

and

$$
\begin{aligned}
\max _{x \in\left(\overline{\left.B_{r}(0) \backslash B_{r / 2}(0)\right) \cap V}\right.} I(\tilde{h}(x)) & \leq \max _{t \geq 0} \max _{y \in \partial B_{r}(0) \cap V} I\left(A_{h}(t, y)\right) \\
& \leq \max _{y \in \partial B_{r}(0) \cap V} I(y) \leq \alpha .
\end{aligned}
$$


As an immediate corollary, we may argue as in the proof of Proposition 3.2 to get

Corollary 4.3. Suppose that I satisfies (LL) and (SP1-3). Then there is a (PS) sequence $\left(u_{n}\right)$ such that $I\left(u_{n}\right) \rightarrow c$.

The disadvantage of our proof is the extra regularity assumption (LL). An advantage is the more constructive nature, analogous to the procedure for the MPL:

Step 1: Pick an $h \in \Gamma_{1}$, and set $i=1$.

Step 2: Deform $h$ using the negative gradient flow to $\psi_{i}(h(\cdot))$.

Step 3: Find $s_{i} \in \overline{B_{r}(0)} \cap V$ so that $I\left(\psi_{i}\left(h\left(s_{i}\right)\right)\right)=\max _{s \in \overline{B_{r}(0)} \cap V} I\left(\psi_{i}(h(s))\right)$.

Step 4: Increment $i$, and then return to step 2 .

By keeping tracking of the "high" points $\psi_{i}\left(h\left(s_{i}\right)\right)$, we get a sequence of points that (under suitable assumptions on $I$ ) converge to a critical point at level higher than $c$. We return to the question of convergence in Sects. 6 and 7 .

\section{A linking type theorem}

Suppose now that $I \in C^{1}(E, \mathbb{R})$ satisfies

(L1) $E=V \oplus X$, where $V$ is finite dimensional

(L2) there is a $\rho>0$ and $\alpha>0$ such that $\left.I\right|_{\partial B_{\rho}(0) \cap X} \geq \alpha$

(L3) there is an $e \in \partial B_{1}(0) \cap X$ and an $R>\rho$ such that if

$$
Q:=\left(B_{R}(0) \cap V\right) \oplus\{r e: 0<r<R\} \text {, then }\left.I\right|_{\partial Q} \leq 0
$$

Here, $\partial Q$ refers to the boundary of $Q$ relative to $V \oplus \operatorname{span}(e)$. Again, Willem showed in [18] that if $I$ satisfies (L1-L3), then $I$ has a (PS) sequence $\left(x_{n}\right)$ such that $I\left(x_{n}\right) \rightarrow c$, where

$$
c:=\inf _{h \in \Gamma} \max _{s \in \bar{Q}} I(h(s)),
$$

and

$$
\Gamma:=\left\{h \in C(\bar{Q}, E):\left.h\right|_{\partial Q}=\mathrm{id}\right\} .
$$

Similar to the case of the saddle point theorem, we define

$$
\begin{aligned}
\Gamma_{1}=\{h \in C(\bar{Q}, E): & \text { there exists } A_{h} \in C([0,1] \times \bar{Q}, E) \\
& \text { satisfying properties (a), (b), and (c) }\},
\end{aligned}
$$

where

(a) $A_{h}(0, x)=x$ for all $x \in \partial Q$,

(b) $A_{h}(1, x)=h(x)$ for all $x \in \bar{Q}$,

(c) For each $x \in \partial Q, t \mapsto I\left(A_{h}(t, x)\right)$ is non-increasing.

We then define

$$
c_{1}=\inf _{h \in \Gamma_{1}} \max _{x \in \bar{Q}} I(h(x)) .
$$


Theorem 5.1. Suppose $I \in C^{1}(E, \mathbb{R})$ satisfies $(L L)$ and (L1-3). Then $c_{1} \geq \alpha$. Moreover, for any $h \in \Gamma_{1}$, there is an $s^{\star} \in \bar{Q}$ and a sequence $\left(t_{n}\right)$ with $t_{n} \rightarrow \infty$ such that if $u_{n}:=\varphi_{t_{n}}\left(h\left(s^{\star}\right)\right)$, then $\left(u_{n}\right)$ is a (PS) sequence and $I\left(u_{n}\right) \geq c_{1}$.

Proof. The proof that $c_{1} \geq \alpha$ is similar to the corresponding argument in Theorem 4.1, and may be found in $[9,14]$ or [18]. The remainder of the proof is very similar to the proof of Theorem 4.1. A key step is establishing that $\Gamma_{1}$ is invariant under the flow $\psi_{t}$ for $t \geq 0$. Given $h \in \Gamma_{1}$ and its corresponding $A_{h}$ and $i \in \mathbb{N}$, we use the same definition of $\Lambda$ with (replacing $\overline{B_{r}(0)} \cap V$ with $\bar{Q})$ as in the proof of Theorem 4.1 .

Next, as with the Saddle Point Theorem, the following proposition implies that $c_{1}=c$.

Proposition 5.2. For any $h \in \Gamma_{1}$, there is a $\tilde{h} \in \Gamma$ such that

$$
\max _{s \in \bar{Q}} I(\tilde{h}(s))=\max _{x \in \bar{Q}} I(h(x)) .
$$

Proof. Let $h \in \Gamma_{1}$ be fixed. Note that $\bar{Q}$ is a closed, convex subset of a finite dimensional vector space. Let $p \in Q^{\circ}$ (the interior relative to $\left.V \oplus \operatorname{span}(e)\right)$, and let $F: \bar{Q} \rightarrow \bar{Q}$ be given by $F(x)=\frac{1}{2}(x+p)$ (since $\bar{Q}$ is convex, $\left.F(x) \in \bar{Q}\right)$. Let $Q^{\prime}:=\operatorname{Im}(F)$, and note that $F$ is a homeomorphism from $\bar{Q}$ to $Q^{\prime}$, with inverse $F^{-1}(x)=2 x-p$. Moreover, $Q^{\prime}$ is convex and $F(\partial \bar{Q})=\partial Q^{\prime}$. We next define a map $\Pi: \bar{Q} \backslash\{p\} \rightarrow \partial \bar{Q}$ as follows: since $\bar{Q}$ is convex, for any $x \in \bar{Q} \backslash\{p\}$, the ray $r_{x}(t)=p+t(x-p)$ for $t>0$ (connecting $p$ to $x$ ) crosses $\partial \bar{Q}$ at exactly one point. This unique point is $\Pi(x)$. Note that for $x \in \partial Q^{\prime}, \Pi(x)=2 x-p=F^{-1}(x)$ and for $x \in \partial \bar{Q}, \Pi(x)=x$. Let

$$
f(x)=\frac{\operatorname{dist}\left(x, \bar{Q}^{c}\right)}{\operatorname{dist}\left(x, \bar{Q}^{c}\right)+\operatorname{dist}\left(x, Q^{\prime}\right)},
$$

and define $\tilde{h}$ by:

$$
\tilde{h}(x):= \begin{cases}h\left(F^{-1}(x)\right) & \text { if } x \in Q^{\prime} \\ A_{h}(f(x), \Pi(x)) & \text { if } x \in \bar{Q} \backslash Q^{\prime} .\end{cases}
$$

Note that as $x \in \bar{Q} \backslash Q^{\prime}$ approaches $\partial Q^{\prime}, \Pi(x) \rightarrow F^{-1}(x)$ and $f(x) \rightarrow 1$. Thus, $A_{h}(f(x), \Pi(x)) \rightarrow A_{h}\left(1, F^{-1}(x)\right)=h\left(F^{-1}(x)\right)$ and so $\tilde{h} \in C(\bar{Q}, E)$. Next, if $x \in \partial Q, f(x)=0$ and so $\tilde{h}(x)=A_{h}(0, \Pi(x))=\Pi(x)=x$, since $\Pi$ fixed the boundary of $\bar{Q}$. Therefore, $\tilde{h} \in \Gamma$.

The proof then follows from the following straightforward consequences of the definition:

$$
\max _{s \in Q^{\prime}} I(\tilde{h}(s))=\max _{x \in \bar{Q}} I(h(x)) \geq \alpha
$$

and

$$
\max _{x \in \bar{Q} \backslash Q^{\prime}} I(\tilde{h}(x)) \leq \max _{t \in[0,1]} \max _{y \in \partial Q} I\left(A_{h}(t, y)\right) \leq \max _{y \in \partial Q} I(y) \leq 0,
$$

where we have used the fact that if $x \in \bar{Q} \backslash Q^{\prime}$, then $\Pi(x) \in \partial Q$. Thus, for $x \in \bar{Q} \backslash Q^{\prime}, \tilde{h}(x)=A_{h}(t, y)$ for some $t \in[0,1]$ and $y \in \partial Q$. 
As for Theorem 4.1, we immediately have the following:

Corollary 5.3. Suppose that I satisfies (LL) and (L1-3). Then there is a (PS) sequence $u_{n}$ such that $I\left(u_{n}\right) \rightarrow c$.

There is an analogous algorithm for this type of linking theorem as there is for the saddle point. In the next two sections, we address the question of convergence of the sequence of high points to a critical point.

\section{Convergence}

We henceforth assume that $I$ satisfies(L1-3), (LL), the Palais-Smale condition, and that the critical points of $I$ are isolated. Note that this implies that for any $a<b$, the set $\mathcal{K}_{a}^{b}=\{u \in \mathcal{K}: a \leq I(u) \leq b\}$ is finite.

In the proof of Theorems 3.1, 4.1, and 5.1, the following process was used:

Step 1: Pick an $h \in \Gamma_{1}$, and set $i=1$.

Step 2: Deform $h$ using the negative gradient flow to $\psi_{i}(h(\cdot))$.

Step 3: Find $s_{i}$ that maximizes $I\left(\psi_{i}(h(s))\right)$ over the deformed set.

Step 4: Increment $i$, and then return to step 2 .

This process creates a sequence of "high" points $s_{i}$. Because of compactness of the set from which $s_{i}$ is picked, a subsequence of $s_{i}$ converged to some $s^{\star}$, and some subsequence of $\psi_{t}\left(h\left(s^{\star}\right)\right)$ was a (PS) sequence for $I$. Our goal in the following two sections is to show

1. $\psi_{t}\left(s^{\star}\right)$ converges as $t \rightarrow \infty$ to some $u \in \mathcal{K}$, and

2. that a subsequence of high points $\psi_{i}\left(h\left(s_{i}\right)\right) \rightarrow u$.

To do this, we need the following lemmas. The first says that for any $x, \psi_{t}(x)$ has a maximum speed of 1 and the second says that if $\psi_{t}(x)$ spends some interval of time away $\mathcal{K}$, then $I\left(\psi_{t}(x)\right)$ decreases by at least some fixed amount on that interval. In what follows, $I_{a}^{b}:=\{x \in E: a \leq I(x) \leq b\}$. Since $I$ satisfies the (PS) condition, there is a critical value $\hat{c}$ provided by Theorem 5.1.

Lemma 6.1. For any $x \in E$ and any $t, s \geq 0,\left\|\psi_{t}(x)-\psi_{s}(x)\right\| \leq|t-s|$.

Proof. This is clearly true if $x \in \mathcal{K}$. Suppose now that $x \notin \mathcal{K}$. Supposing that $t>s$, we have by $(2.1)$

$$
\begin{aligned}
\left\|\psi_{t}(x)-\psi_{s}(x)\right\|=\left\|\varphi_{t}(x)-\varphi_{s}(x)\right\| & =\left\|\int_{s}^{t} \frac{d}{d t} \varphi_{t}(x) d t\right\| \\
& \leq \int_{s}^{t}\left\|\frac{V\left(\varphi_{t}(x)\right)}{1+\left\|V\left(\varphi_{t}(x)\right)\right\|}\right\| d t \\
& =\int_{s}^{t} \frac{\left\|V\left(\varphi_{t}(x)\right)\right\|}{1+\left\|V\left(\varphi_{t}(x)\right)\right\|} d t \\
& \leq t-s,
\end{aligned}
$$

as desired.

Lemma 6.2. Suppose there is a $\delta>0$ such that $\left\|I^{\prime}(y)\right\| \geq \delta$ for all $y \in A \subseteq E$. If $\psi_{t}(x) \in A$ for all $t \in[a, b]$, then $I\left(\psi_{b}(x)\right) \leq I\left(\psi_{a}(x)\right)-\frac{\delta^{2}}{4+4 \delta}(b-a)$. 
Proof. Note that $A$ and $\mathcal{K}$ are disjoint, and so if $\psi_{t}(x) \in A$, then $\psi_{t}(x)=\varphi_{t}(x)$. Therefore, by (2.1) we have

$$
\begin{aligned}
I\left(\varphi_{b}(x)\right)-I\left(\varphi_{a}(x)\right) & =\int_{a}^{b} \frac{d}{d t} I\left(\varphi_{t}(x)\right) d t \\
& =\int_{a}^{b}-\frac{1}{1+\left\|V\left(\varphi_{t}(x)\right)\right\|} I^{\prime}\left(\varphi_{t}(x)\right) V\left(\varphi_{t}(x)\right) d t \\
& \leq \int_{a}^{b}-\frac{1}{1+\left\|V\left(\varphi_{t}(x)\right)\right\|}\left\|I^{\prime}\left(\varphi_{t}(x)\right)\right\|^{2} d t,
\end{aligned}
$$

where we have used (PG2). Note that (PG1) implies that $\left\|I^{\prime}(x)\right\| \leq\|V(x)\| \leq$ $2\left\|I^{\prime}(x)\right\|$ and so $\frac{1}{4}\left\|V\left(\varphi_{t}(x)\right)\right\|^{2} \leq\left\|I^{\prime}\left(\varphi_{t}(x)\right)\right\|^{2}$. Substituting this inequality into (6.1) gives

$$
\begin{aligned}
I\left(\varphi_{b}(x)\right)-I\left(\varphi_{a}(x)\right) & \leq-\frac{1}{4} \int_{a}^{b} \frac{\left\|V\left(\varphi_{t}(x)\right)\right\|^{2}}{1+\left\|V\left(\varphi_{t}(x)\right)\right\|} \\
& =-\frac{1}{4} \int_{a}^{b} f\left(\left\|V\left(\varphi_{t}(x)\right)\right\|\right) d t
\end{aligned}
$$

where $f(z):=\frac{z^{2}}{1+z}$. Note that $f$ is increasing for $z \geq 0$. Since $\left\|I^{\prime}\left(\varphi_{t}(x)\right)\right\| \leq$ $\left\|V\left(\varphi_{t}(x)\right)\right\|$ by (PG2) and $\delta \leq\left\|I^{\prime}\left(\varphi_{t}(x)\right)\right\|$ by assumption, we then have

$$
I\left(\varphi_{b}(x)\right)-I\left(\varphi_{a}(x)\right) \leq-\frac{1}{4} \int_{a}^{b} f(\delta) d t=-\frac{\delta^{2}}{4+4 \delta}(b-a),
$$

and so

$$
I\left(\varphi_{a}(x)\right)-\frac{\delta^{2}}{4+4 \delta}(b-a) \geq I\left(\varphi_{b}(x)\right),
$$

as desired.

Combining the previous lemmas, we have the following

Corollary 6.3. Suppose that there is a $\delta>0$ such that $\left\|I^{\prime}(y)\right\| \geq \delta$ for all $y \in A$. If $\left\|\psi_{b}(x)-\psi_{a}(x)\right\| \geq \eta$ and $\psi_{t}(x) \in A$ for all $t \in[a, b]$, then $I\left(\psi_{b}(x)\right) \leq$ $I\left(\psi_{a}(x)\right)-\frac{\delta^{2}}{4+4 \delta} \cdot \eta$.

Corollary 6.3 implies that as $\psi_{t}(x)$ transitions between neighborhoods of $\mathcal{K}_{b}^{a}$, the value of $I$ along the flow line must decrease by at least some amount. Because we assume that $I$ satisfies (PS), for all $\eta>0$, we know that

$$
\delta(\eta, c, d):=\inf \left\{\left\|I^{\prime}(z)\right\|: z \in I_{c}^{d} \backslash N_{\eta}(\mathcal{K})\right\}>0,
$$

where $N_{\eta}(A):=\bigcup_{x \in A} B_{\eta}(x)$.

We now show that $\psi_{t}\left(h\left(s^{\star}\right)\right) \rightarrow u \in \mathcal{K}$ as $t \rightarrow \infty$. First, we show that $\psi_{t}\left(h\left(s^{\star}\right)\right)$ is bounded, and then we show that $I^{\prime}\left(\psi_{t}\left(h\left(s^{\star}\right)\right)\right) \rightarrow 0$ as $t \rightarrow \infty$. 
Proposition 6.4. $\psi_{t}\left(h\left(s^{\star}\right)\right)$ is bounded in E.

Proof. This is obvious if $h\left(s^{\star}\right) \in \mathcal{K}$. Suppose then that $h\left(s^{\star}\right) \notin \mathcal{K}$. Then $\psi_{t}\left(h\left(s^{\star}\right)\right)=\varphi_{t}\left(h\left(s^{\star}\right)\right)$. From Theorem 5.1 , there is a sequence $\left(t_{j}\right)$ with $t_{j} \rightarrow \infty$ such that $I^{\prime}\left(\varphi_{t_{j}}\left(h\left(s^{\star}\right)\right)\right) \rightarrow 0$ and $I\left(\varphi_{t_{j}}\left(h\left(s^{\star}\right)\right)\right)$ is bounded. Since $I$ satisfies (PS), passing to a subsequence, we may assume $\varphi_{t_{j}}\left(h\left(s^{\star}\right)\right) \rightarrow u \in \mathcal{K}_{\alpha}^{M}$, where $M=\max _{s \in \bar{Q}} I(h(s))$. Therefore, for all sufficiently large $j, \varphi_{t_{j}}\left(h\left(s^{\star}\right)\right) \in N_{1}\left(\mathcal{K}_{\alpha}^{M}\right)$.

Because $I$ satisfies the (PS) condition, $\mathcal{K}_{\alpha}^{M}$ is compact. Thus, if $\varphi_{t}\left(h\left(s^{\star}\right)\right)$ is unbounded in $t$, then there exists a sequence $\left(\tau_{j}\right)$ such that $\tau_{j} \rightarrow \infty$ and $\varphi_{\tau_{j}}\left(h\left(s^{\star}\right)\right)$ gets farther and farther from $N_{1}\left(\mathcal{K}_{\alpha}^{M}\right)$. Taking $A:=I_{\alpha}^{M} \backslash N_{1}\left(\mathcal{K}_{\alpha}^{M}\right)$ and $\delta:=\delta(1, \alpha, M)$ from (6.2), Corollary 6.3 then implies that $I\left(\varphi_{t}\left(h\left(s^{\star}\right)\right)\right) \rightarrow$ $-\infty$, which is a contradiction.

Proposition 6.5. $I^{\prime}\left(\psi_{t}\left(h\left(s^{\star}\right)\right)\right) \rightarrow 0$ as $t \rightarrow \infty$.

Proof. Again, this is obvious if $h\left(s^{\star}\right) \in \mathcal{K}$. Suppose then that $h\left(s^{\star}\right) \notin \mathcal{K}$. Then $\psi_{t}\left(h\left(s^{\star}\right)\right)=\varphi_{t}\left(h\left(s^{\star}\right)\right)$. From the proof of Theorem 5.1, we know that $\int_{0}^{\infty} \frac{\left\|V\left(\varphi_{t}\left(h\left(s^{\star}\right)\right)\right)\right\|^{2}}{1+\left\|V\left(\varphi_{t}\left(h\left(s^{\star}\right)\right)\right)\right\|} d t<\infty$. Let $f(z)=\frac{z^{2}}{1+z}$, and note that $f$ is increasing for $z \geq 0$. (PG2) implies that $\left\|I^{\prime}\left(\varphi_{t}(x)\right)\right\| \leq\left\|V\left(\varphi_{t}(x)\right)\right\|$ and it follows that $\int_{0}^{\infty} f\left(\left\|I^{\prime}\left(\varphi_{t}\left(h\left(s^{\star}\right)\right)\right)\right\|\right) d t<\infty$. Since $f(z) \rightarrow 0$ if and only if $z \rightarrow 0$, to show that $I^{\prime}\left(\varphi_{t}\left(h\left(s^{\star}\right)\right)\right) \rightarrow 0$, it suffices to show that $f\left(\left\|I^{\prime}\left(\varphi_{t}\left(h\left(s^{\star}\right)\right)\right)\right\|\right) \rightarrow 0$. Since $\int_{0}^{\infty} f\left(\left\|I^{\prime}\left(\varphi_{t}\left(h\left(s^{\star}\right)\right)\right)\right\|\right) d t<\infty$, it suffices to show that $f\left(\left\|I^{\prime}\left(\varphi_{t}\left(h\left(s^{\star}\right)\right)\right)\right\|\right)$ is uniformly continuous in $t$. Note that since $f^{\prime}(z)$ is bounded for $z \geq 0, f$ : $(0, \infty) \rightarrow(0, \infty)$ is Lipschitz, and so we need only show that $I^{\prime}\left(\varphi_{t}\left(h\left(s^{\star}\right)\right)\right)$ is a uniformly continuous function of $t$. By (LL) and the assumption that $I$ satisfies (PS), there is an $\eta>0$ such that $I^{\prime}$ is Lipschitz on $N_{\eta}\left(\mathcal{K}_{\alpha}^{M}\right)$. Since Lemma 6.1 implies that $\varphi_{t}\left(h\left(s^{\star}\right)\right)$ is a Lipschitz function of $t$, if we can show that there is a $T>0$ such that $\varphi_{t}\left(h\left(s^{\star}\right)\right) \in N_{\eta}\left(\mathcal{K}_{\alpha}^{M}\right)$ for all $t>T$, we will know that $I^{\prime}\left(\varphi_{t}\left(h\left(s^{\star}\right)\right)\right)$ is a uniformly continuous function of $t$.

Suppose then that there is no such $T$. Since $\varphi_{t_{j}}\left(h\left(s^{\star}\right)\right) \rightarrow u \in \mathcal{K}_{\alpha}^{m}$, for all sufficiently large $j, \varphi_{t_{j}}\left(h\left(s^{\star}\right)\right) \in N_{\eta / 2}\left(\mathcal{K}_{\alpha}^{M}\right)$. Since there is no appropriate $T$, there must a sequence $\left(s_{j}\right)$ such that $\varphi_{s_{j}}\left(h\left(s^{\star}\right)\right) \notin N_{\eta}\left(\mathcal{K}_{\alpha}^{M}\right)$. By picking appropriate subsequences, we may assume $s_{j}<t_{j}<s_{j+1}<t_{j+1}$. Note that there must be a sub-interval $\left(a_{j}, b_{j}\right) \subseteq\left(s_{j}, t_{j}\right)$ so that for $t \in$ $\left(a_{j}, b_{j}\right), \varphi_{t}\left(h\left(s^{\star}\right)\right) \notin N_{\eta / 2}\left(\mathcal{K}_{\alpha}^{M}\right)$. This implies that $\left\|\varphi_{a_{j}}\left(h\left(s^{\star}\right)\right)-\varphi_{b_{j}}\left(h\left(s^{\star}\right)\right)\right\| \geq$ $\eta / 2$. Taking $A=I_{\alpha}^{M} \backslash N_{\eta / 2}\left(\mathcal{K}_{\alpha}^{M}\right)$ and $\delta=\delta(\eta / 2, \alpha, M)$, Corollary 6.3 would imply that $I\left(\varphi_{t}\left(h\left(s^{\star}\right)\right)\right) \rightarrow-\infty$.

Proposition 6.6. Suppose that $\left(t_{j}\right)$ is a sequence such that $t_{j} \rightarrow \infty$ and $\psi_{t_{j}}\left(h\left(s^{\star}\right)\right) \rightarrow u$ as $j \rightarrow \infty$. Then, in fact $\psi_{t}\left(h\left(s^{\star}\right)\right) \rightarrow u$ as $t \rightarrow \infty$.

Proof. This is clear if $h\left(s^{\star}\right) \in \mathcal{K}$. Suppose then that $h\left(s^{\star}\right) \notin \mathcal{K}$, in which case $\psi_{t}\left(h\left(s^{\star}\right)\right)=\varphi_{t}\left(h\left(s^{\star}\right)\right)$. If Prop 6.6 were not true, there must be an $\eta>0$ and a sequence $\left(s_{j}\right)$ such that $s_{j} \rightarrow \infty$ and $\varphi_{s_{j}}\left(h\left(s^{\star}\right)\right) \notin B_{\eta}(u)$. Note that since $I^{\prime}\left(\varphi_{t}\left(h\left(s^{\star}\right)\right)\right) \rightarrow 0,\left(\varphi_{s_{j}}\left(h\left(s^{\star}\right)\right)\right)$ is a (PS) sequence, and so passing to a subsequence, there must be a $\hat{u} \neq u$ such that $\varphi_{s_{j}}\left(h\left(s^{\star}\right)\right) \rightarrow \hat{u}$ as $j \rightarrow \infty$. Thus, for all large $j$, we must also have $\varphi_{s_{j}}\left(h\left(s^{\star}\right)\right) \in B_{\eta}(\hat{u})$. Since $I$ satisfies (PS) 
and critical points of $I$ in $I_{\alpha}^{M}$ are isolated, by taking $\eta$ smaller if necessary, we may assume that $N_{\eta}\left(\mathcal{K}_{\alpha}^{M}\right)$ is a disjoint union of balls of radius $\eta$ centered at elements of $\mathcal{K}_{\alpha}^{M}$. Passing to subsequences of $\left(s_{j}\right)$ and $\left(t_{j}\right)$, we may assume that $s_{j}<t_{j}<s_{j+1}<t_{j+1}$. Since $N_{\eta}\left(K_{\alpha}^{M}\right)$ is a disjoint collection of balls of radius $\eta$ centered at elements of $\mathcal{K}_{\alpha}^{M}$, in each interval $\left(s_{j}, t_{j}\right)$ there must be a maximal interval $\left(a_{j}, b_{j}\right)$ on which $\varphi_{t}\left(h\left(s^{\star}\right)\right) \notin N_{\eta}\left(\mathcal{K}_{\alpha}^{M}\right)$. Moreover, since there are only finitely many balls making up $N_{\eta}\left(\mathcal{K}_{\alpha}^{M}\right)$, they are all some positive distance $\hat{\eta}$ apart. Thus, since $\varphi_{t}\left(h\left(s^{\star}\right)\right)$ must travel between at least two of them, we may also assume that $\left\|\varphi_{a_{j}}\left(h\left(s^{\star}\right)\right)-\varphi_{b_{j}}\left(h\left(s^{\star}\right)\right)\right\| \geq \hat{\eta}$. Replacing $\eta$ Taking $A=I_{\alpha}^{M} \backslash N_{\eta}\left(\mathcal{K}_{\alpha}^{M}\right), \delta=\delta(\eta, \alpha, M)$ from (6.2) and $\eta=\hat{\eta}$, Lemma 6.3 would imply that $I\left(\varphi_{t}\left(h\left(s^{\star}\right)\right)\right) \rightarrow-\infty$.

\section{Approximation}

In the proof of Theorem 5.1, $s^{\star}$ was found as the limit of a subsequence of $\left(s_{i}\right)$, and each $s_{i}$ was picked so that $\psi_{i}\left(h\left(s_{i}\right)\right)$ maximizes $I$ on the image of the map $s \mapsto \psi_{i}(h(s))$. Thus $\psi_{i}\left(h\left(s_{i}\right)\right)$ is a sequence of high points as $h(\bar{Q})$ is deformed. The goal of this section is to prove that if $I$ satisfies (LL), (L1-3), (PS), critical points of $I$ are isolated and $s_{i_{j}} \rightarrow s^{\star}$, then $\psi_{i_{j}}\left(h\left(s_{i_{j}}\right)\right) \rightarrow u=\lim _{t \rightarrow \infty} \psi_{t}\left(h\left(s^{\star}\right)\right)$, i.e. along a subsequence, the high points converge to the same critical point as $\psi_{t}\left(h\left(s^{\star}\right)\right)$. First, we show that elevation of the high points converges to the same elevation.

Lemma 7.1. $I\left(\psi_{i}\left(h\left(s_{i}\right)\right)\right) \rightarrow I(u)$ as $i \rightarrow \infty$.

Proof. By Lemma 2.3,

$$
I\left(\psi_{i+1}\left(h\left(s_{i+1}\right)\right)\right)=\max _{s \in \bar{Q}} I\left(\psi_{i+1}(h(s))\right) \leq \max _{s \in \bar{Q}} I\left(\psi_{i}(h(s))\right)=I\left(\psi_{i}\left(h\left(s_{i}\right)\right)\right)
$$

and so $I\left(\psi_{i}\left(h\left(s_{i}\right)\right)\right)$ is a decreasing sequence. Moreover, the choice of $s_{i}$ implies that $I\left(\psi_{i}\left(s^{\star}\right)\right) \leq I\left(\psi_{i}\left(h\left(s_{i}\right)\right)\right)$. Since $\psi_{t}\left(h\left(s^{\star}\right)\right) \rightarrow u$ by Proposition 6.6, Lemma 2.3 also implies $I(u) \leq I\left(\psi_{i}\left(h\left(s_{i}\right)\right)\right)$. Therefore, to finish the proof, we need only to show that for any $\varepsilon>0$, there is an $i$ such that $I\left(\psi_{i}\left(h\left(s_{i}\right)\right)\right) \leq I(u)+\varepsilon$.

Let $\varepsilon>0$ be given. Since $\psi_{t}\left(h\left(s^{\star}\right)\right) \rightarrow u$, there is an $i_{0}$ such that $I\left(\psi_{i_{0}}\left(h\left(s^{\star}\right)\right)\right) \leq I(u)+\frac{\varepsilon}{2}$. Since $s_{i_{j}} \rightarrow s^{\star}$, the continuity of the flow and $h$ implies that there is a $i_{j_{1}}>i_{0}$ such that $I\left(\psi_{i_{0}}\left(h\left(s_{i_{j_{1}}}\right)\right)\right)<I(u)+\varepsilon$. But then Lemma 2.3 implies

$$
I\left(\psi_{i_{j_{1}}}\left(h\left(s_{i_{j_{1}}}\right)\right)\right)=I\left(\psi_{i_{j_{1}}-i_{0}}\left(\psi_{i_{0}}\left(h\left(s_{i_{j_{1}}}\right)\right)\right)\right) \leq I\left(\psi_{i_{0}}\left(h\left(s_{i_{j_{1}}}\right)\right)\right)<I(u)+\varepsilon .
$$

Proposition 7.2. Suppose that $\left(x_{n}\right)$ is a sequence such that $x_{n} \rightarrow x^{\star}, \psi_{t}\left(x^{\star}\right) \rightarrow$ $u \in \mathcal{K}$ as $t \rightarrow \infty$ and $I\left(\psi_{n}\left(x_{n}\right)\right)$ is a decreasing sequence that converges to $I(u)$. Then $\left(\psi_{n}\left(x_{n}\right)\right)$ is a $(P S)$ sequence for $I$. 
Proof. Suppose not. Since $I\left(\psi_{n}\left(x_{n}\right)\right)$ is bounded, there must be a subsequence $\left(x_{n_{j}}\right)$ and a $\beta>0$ such that

$$
\left\|I^{\prime}\left(\psi_{n_{j}}\left(x_{n_{j}}\right)\right)\right\| \geq \beta \quad \text { for all } j .
$$

Let $\hat{c}:=I(u)$. Since $I^{\prime}$ is continuous on the compact set $\mathcal{K}_{\hat{c}}^{M}$, there is an $\eta>0$ such that $\left\|I^{\prime}(x)\right\|<\beta$ whenever $x \in N_{\eta}\left(\mathcal{K}_{\hat{c}}^{M}\right)$. Thus, we must have

$$
\psi_{n_{j}}\left(x_{n_{j}}\right) \notin N_{\eta}\left(\mathcal{K}_{\hat{c}}^{M}\right) \quad \text { for all } j .
$$

Since $\psi_{t}\left(x^{\star}\right) \rightarrow u$, there is a $T$ such that if $t \geq T$, then

$$
\left\|\psi_{t}\left(x^{\star}\right)-u\right\|<\frac{\eta}{3} .
$$

Since $x_{n_{j}} \rightarrow x^{\star}$, there is a $J(T)$ such that

$$
\left\|\psi_{T}\left(x_{n_{j}}\right)-u\right\|<\frac{\eta}{2} \quad \text { whenever } j \geq J(T) .
$$

Taking $J(T)$ larger if necessary, we may assume that $n_{J}>T$. Thus, for all $j>J(T)$, we will have

$$
\psi_{T}\left(x_{n_{j}}\right) \in N_{\eta / 2}\left(\mathcal{K}_{\hat{c}}^{M}\right) \quad \text { and } \quad \psi_{n_{j}}\left(x_{n_{j}}\right) \notin N_{\eta}\left(\mathcal{K}_{\hat{c}}^{M}\right)
$$

Let

$$
a_{j}:=\inf \left\{\tau \leq n_{j}: \psi_{t}\left(x_{n_{j}}\right) \notin N_{\eta / 2}\left(\mathcal{K}_{\hat{c}}^{M}\right) \text { for all } t \in\left(\tau, n_{j}\right)\right\} .
$$

Note that $a_{j}>T$, since $\psi_{T}\left(x_{n_{j}}\right) \in B_{\eta / 2}\left(\mathcal{K}_{\hat{c}}^{M}\right) \subseteq N_{\eta / 2}\left(\mathcal{K}_{\hat{c}}^{M}\right)$. Moreover,

- $\psi_{a_{j}}\left(x_{n_{j}}\right) \in \partial N_{\eta / 2}\left(\mathcal{K}_{\hat{c}}^{M}\right)$

- $\left\|\psi_{a_{j}}\left(x_{n_{j}}\right)-\psi_{n_{j}}\left(x_{n_{j}}\right)\right\| \geq \frac{\eta}{2}$

- $\psi_{t}\left(x_{n_{j}}\right) \notin N_{\eta / 2}\left(\mathcal{K}_{\hat{c}}^{M}\right)$ for all $t \in\left[a_{j}, n_{j}\right]$.

Since $I$ satisfies (PS), if $\delta:=\delta(\eta / 2, M, \hat{c})$ and $A:=I_{\hat{c}}^{M} \backslash N_{\eta / 2}\left(\mathcal{K}_{\hat{c}}^{M}\right)$, Corollary 6.3 implies that

$$
I\left(\psi_{n_{j}}\left(x_{n_{j}}\right)\right) \leq I\left(\psi_{a_{j}}\left(x_{n_{j}}\right)\right)-\frac{\delta^{2}}{4+4 \delta} \cdot \frac{\eta}{2} .
$$

Let $\alpha:=\frac{\delta^{2}}{4+4 \delta}$. Since $I\left(\psi_{n}\left(x_{n}\right)\right)$ decreases to $I(u)=\hat{c}$, we have

$$
\hat{c}+\alpha \cdot \frac{\eta}{2} \leq I\left(\psi_{a_{j}}\left(x_{n_{j}}\right)\right) \quad \text { for all } j>J(T) .
$$

Since $a_{j}>T$, Lemma 2.3 implies

$$
\hat{c}+\frac{\alpha \cdot \eta}{2} \leq I\left(\psi_{T}\left(x_{n_{j}}\right)\right) \quad \text { for all } j>J(T) .
$$

Notice that the left side depends only on $\hat{c}, \delta$ and $\eta$, which are all independent of $T, j$ and $J(T)$, we may let $j \rightarrow \infty$ to get

$$
\hat{c}+\frac{\alpha \cdot \eta}{2} \leq I\left(\psi_{T}\left(x^{\star}\right)\right) .
$$

Letting now $T \rightarrow \infty$, we will have

$$
\hat{c}+\frac{\alpha \cdot \eta}{2} \leq I(u)=\hat{c},
$$

which is impossible. 
Theorem 7.3. Suppose that $\left(x_{n}\right)$ is a sequence such that $x_{n} \rightarrow x^{\star}, \psi_{t}\left(x^{\star}\right) \rightarrow$ $u \in \mathcal{K}$ as $t \rightarrow \infty$ and $I\left(\psi_{n}\left(x_{n}\right)\right)$ is a decreasing sequence that converges to $I(u)$. Then $\psi_{n}\left(x_{n}\right) \rightarrow u$.

Proof. Suppose that this is false. Then there is a subsequence $\left(\psi_{n_{j}}\left(x_{n_{j}}\right)\right)$ and an $\eta>0$ such that $\left\|\psi_{n_{j}}\left(x_{n_{j}}\right)-u\right\| \geq \eta$. Let $\hat{c}:=I(u)$. Since we've assumed that critical points of $I$ are isolated, by taking $\eta$ smaller if necessary, we may assume that $N_{\eta}\left(\mathcal{K}_{\hat{c}}^{M}\right)$ is a disjoint union of balls of radius $\eta$. Moreover, we may assume that the distance between balls is at least $\eta$. By Proposition $7.2, \psi_{n_{j}}\left(x_{n_{j}}\right)$ is a (PS) sequence, and so passing to a relabeled subsequence, $\psi_{n_{j}}\left(x_{n_{j}}\right) \rightarrow \hat{u}, u \neq \hat{u}$. Moreover, we may assume that $\left\|\psi_{n_{j}}\left(x_{n_{j}}\right)-\hat{u}\right\|<\frac{\eta}{2}$ for all $j$. Since $\psi_{t}\left(x^{\star}\right) \rightarrow u$, there is at $T$ such that $\left\|\psi_{t}\left(x^{\star}\right)-u\right\|<\frac{\eta}{2}$ for all $t \geq T$. Since $x_{n_{j}} \rightarrow x^{\star}$, there is a $J(T)$ such that if $j>J(T)$, then $\left\|\psi_{T}\left(x_{n_{j}}\right)-u\right\|<\eta$. By taking $J(T)$ larger if necessary, we may assume that $T<n_{J(T)}$, and so $T<n_{j}$ whenever $j>J(T)$.

On the interval $\left(T, n_{j}\right), \psi_{t}\left(x_{n_{j}}\right)$ must travel from $B_{\eta}(u)$ (at $t=T$ ) to $B_{\eta / 2}(\hat{u})\left(\right.$ at $\left.t=n_{j}\right)$. Let

$$
a_{j}:=\inf \left\{\tau: \psi_{t}\left(x_{n_{j}}\right) \in B_{\eta}(\hat{u}) \text { for all } t \in\left(\tau, n_{j}\right)\right\} .
$$

Note that $\psi_{a_{j}}\left(x_{n_{j}}\right) \in \partial B_{\eta}(\hat{u})$ and $\psi_{t}\left(x_{n_{j}}\right) \in B_{\eta}(\hat{u})$ for all $t \in\left(a_{j}, n_{j}\right)$. Next, let

$$
b_{j}:=\sup \left\{\tau: \psi_{t}\left(x_{n_{j}}\right) \in B_{\eta}(\hat{u}) \backslash B_{\eta / 2}(\hat{u}) \text { for all } t \in\left(a_{j}, \tau\right)\right\} .
$$

Note that $\psi_{b_{j}}\left(x_{n_{j}}\right) \in \partial B_{\eta / 2}(\hat{u})$, and so $\left\|\psi_{a_{j}}\left(x_{n_{j}}\right)-\psi_{b_{j}}\left(x_{n_{j}}\right)\right\| \geq \frac{\eta}{2}$. Moreover, $\psi_{t}\left(x_{n_{j}}\right) \notin N_{\eta / 2}\left(\mathcal{K}_{\hat{c}}^{M}\right)$ for all $t \in\left(a_{j}, b_{j}\right)$. Thus, by Corollary 6.3 , if we take $A=I_{\hat{c}}^{M} \backslash N_{\eta / 2}\left(\mathcal{K}_{\hat{c}}^{M}\right), \delta=\delta(\eta / 2, \hat{c}, M)$ and $\alpha:=\frac{\delta^{2}}{4+4 \delta}$, we then have

$$
I\left(\psi_{b_{j}}\left(x_{n_{j}}\right)\right) \leq I\left(\psi_{a_{j}}\left(x_{n_{j}}\right)\right)-\alpha \cdot \frac{\eta}{2} \quad \text { for all } j>J(T) .
$$

Lemma 2.3 and (7.2) together imply that

$$
\begin{aligned}
I\left(\psi_{n_{j}}\left(x_{n_{j}}\right)\right) & \leq I\left(\psi_{b_{j}}\left(x_{n_{j}}\right)\right) \leq I\left(\psi_{a_{j}}\left(x_{n_{j}}\right)\right)-\alpha \cdot \frac{\eta}{2} \\
& \leq I\left(\psi_{T}\left(x_{n_{j}}\right)\right)-\frac{1}{2} \text { anforallj }>J(T) .
\end{aligned}
$$

Since $\alpha$ and $\eta$ are independent of $T, J$ and $j$, letting $j \rightarrow \infty$ in (7.3) then implies that

$$
\hat{c} \leq I\left(\psi_{T}\left(x^{\star}\right)\right)-\frac{1}{2} \alpha \eta .
$$

Taking the limit above as $T \rightarrow \infty$, we have

$$
\hat{c} \leq \hat{c}-\frac{1}{2} \alpha \eta
$$

which is impossible.

Taking $x_{n}:=h\left(s_{n}\right)$ and $x^{\star}:=h\left(s^{\star}\right)$, Lemma 7.1 implies that $I\left(\psi_{n}\left(x_{n}\right)\right)$ decreases to $I(u)$, and Proposition 6.6 implies that $\psi_{t}\left(x^{\star}\right) \rightarrow u \in \mathcal{K}$. Therefore, if $I$ satisfies (PS) and (LL) and the critical points of $I$ are isolated, the 
sequence of high points arising from the mountain pass or saddle point or linking do in fact converge to a critical point of $I$. If the critical points of $I$ are not isolated, then the high points will converge to a connected component of the critical set.

\section{Acknowledgements}

My thanks to the referee for their many helpful comments and suggestions, especially their improvements to the proofs of Proposition 7.2 and Theorem 7.3.

\section{References}

[1] Barutello, V., Terracini, S.: A bisection algorithm for the numerical mountain pass. NoDEA 14, 527-539 (2007)

[2] Bisgard, J.: Heteroclinic and homoclinic solutions for two classes of Hamiltonian systems. University of Wisconsin-Madison Thesis (2005)

[3] Bisgard, J.: Homoclinics for a Hamiltonian with wells at different levels. Calc. Var. 29, 1-30 (2007)

[4] Bolotin, S., Rabinowitz, P.H.: On the multiplicity of periodic solutions of mountain pass type for a class of semilinear PDE's. J. Fixed Point Theory Appl. 2, 313-331 (2007)

[5] Chang, K.C.: Heat method in nonlinear elliptic equations, topological methods, variational methods and their applications (Taiyuan, 2002), pp. 65-76. World Sci. Publ., River Edge (2003)

[6] Choi, Y.S., McKenna, P.J.: A mountain pass method for the numerical solution of semilinear elliptic problems. Nonlinear Anal. 20, 417-437 (1993)

[7] Ding, Z., Costa, D., Chen, G.: A high-linking algorithm for sign-changing solutions of semilinear elliptic equations. Nonlinear Anal. 38, 151-172 (1999)

[8] Hofer, H., Zehnder, E.: Periodic solutions on hypersurfaces and a result by C. Viterbo. Invent. Math. 90, 1-9 (1987)

[9] Jabri, Y.: The mountain pass theorem: variants, generalizations and some applications. Cambridge University Press, London (2003)

[10] Lewis, A., Jeffrey Pang, C.H.: Level set methods for finding critical points of mountain pass type. Nonlinear Anal. 74, 4058-4082 (2011)

[11] Li, Y., Zhou, J.: A minimax method for finding multiple critical points and its applications to semilinear PDEs. SIAM J. Sci. Comput. 23, 840-865 (2001)

[12] Li, Y., Zhou, J.: Convergence results of a local minimax method for finding multiple critical points. SIAM J. Sci. Comput. 24, 865-885 (2002)

[13] Mawhin, J., Willem, M.: Origin and evolution of the Palais-Smale condition in critical point theory. J. Fixed Point Theory Appl. 7, 265-290 (2010) 
[14] Rabinowitz, P.H.: Minimax methods in critical point theory with applications to differential equations. AMS (1986)

[15] Rabinowitz, P. H.: Gluing a mountain pass solution to a minimum. Regul. Chaotic Dyn. 14, 163-178 (2009)

[16] Troestler, C., Willem, M.: Nontrivial solution of a semilinear Schrödinger equation. Comm. Partial Differ. Equ. 21, 1431-1449 (1996)

[17] Wang, Z.Q., Zhou, J.: An efficient and stable method for computing multiple saddle points with symmetries. SIAM J. Numer. Anal. 43, 891-907 (2005)

[18] Willem, M.: Minimax theorems. Birkhäuser, Basel (1996)

James Bisgard

Department of Mathematics

Central Washington University

400 E. University Way

Ellensburg

WA 98926-7424

USA

e-mail: bisgardj@cwu.edu

Received: 28 March 2012.

Accepted: 5 January 2013. 